\title{
Insights into the transcriptional responses of a microbial community to silver nanoparticles in a freshwater microcosm ${ }^{\not 3}$
}

\author{
Tao Lu ${ }^{\text {a }}$, Qian Qu ${ }^{\text {a }}$, Michel Lavoie ${ }^{\text {b }}$, Xiangjie Pan ${ }^{\text {c }}$, W.J.G.M. Peijnenburg ${ }^{\text {d, e }}$, \\ Zhigao Zhou ${ }^{a}$, Xiangliang Pan ${ }^{a}$, Zhiqiang Cai ${ }^{\mathrm{f}, * *}$, Haifeng Qian ${ }^{\mathrm{a}, \mathrm{g}, *}$ \\ a College of Environment, Zhejiang University of Technology, Hangzhou, 310032, PR China \\ ${ }^{\mathrm{b}}$ Quebec-Ocean and Takuvik Joint International Research Unit, Université Laval, Québec, G1VOA6, Canada \\ ${ }^{\mathrm{c}}$ Zhejiang Fangyuan Test Group Co Ltd, Hangzhou, 310013, Zhejiang, PR China \\ d Institute of Environmental Sciences (CML), Leiden University, 2300, RA, Leiden, the Netherlands \\ e National Institute of Public Health and the Environment (RIVM), Center for Safety of Substances and Products, P.O. Box 1, 3720, BA, Bilthoven, the \\ Netherlands \\ ${ }^{\mathrm{f}}$ Laboratory of Applied Microbiology and Biotechnology, School of Pharmaceutical Engineering \& Life Science, Changzhou University, Changzhou, Jiangsu, \\ 213164, PR China \\ ${ }^{\mathrm{g}}$ Xinjiang Key Laboratory of Environmental Pollution and Bioremediation, Xinjiang Institute of Ecology and Geography, Chinese Academy of Sciences, \\ Urumqi, 830011, PR China
}

\section{A R T I C L E I N F O}

\section{Article history:}

Received 17 July 2019

Received in revised form 18 November 2019

Accepted 3 December 2019

Available online 5 December 2019

\section{Keywords:}

Cyanobacteria

Green algae

Meta-transcriptome

Eukaryote

Prokaryote

Silver nanoparticles

\begin{abstract}
A B S T R A C T
Silver nanoparticles (AgNPs) are widely used because of their excellent antibacterial properties. They are, however, easily discharged into the water environment, causing potential adverse environmental effects. Meta-transcriptomic analyses are helpful to study the transcriptional response of prokaryotic and eukaryotic aquatic microorganisms to AgNPs. In the present study, microcosms were used to investigate the toxicity of AgNPs to a natural aquatic microbial community. It was found that a 7-day exposure to $10 \mu \mathrm{g} \mathrm{L}^{-1}$ silver nanoparticles (AgNPs) dramatically affected the structure of the microbial community. Aquatic micro eukaryota (including eukaryotic algae, fungi, and zooplankton) and bacteria (i.e., heterotrophic bacteria and cyanobacteria) responded differently to the AgNPs stress. Meta-transcriptomic analyses demonstrated that eukaryota could use multiple cellular strategies to cope with AgNPs stress, such as enhancing nitrogen and sulfur metabolism, over-expressing genes related to translation, amino acids biosynthesis, and promoting bacterial-eukaryotic algae interactions. By contrast, bacteria were negatively affected by AgNPs with less signs of detoxification than in case of eukaryota; various pathways related to energy metabolism, DNA replication and genetic repair were seriously inhibited by AgNPs. As a result, eukaryotic algae (mainly Chlorophyta) dominated over cyanobacteria in the AgNPs treated microcosms over the 7-d exposure. The present study helps to understand the effects of AgNPs on aquatic microorganisms and provides insights into the contrasting AgNPs toxicity in eukaryota and bacteria.
\end{abstract}

(C) 2019 Elsevier Ltd. All rights reserved.

\section{Introduction}

Silver nanoparticles (AgNPs) are widely used in commercial products (Duran et al., 2016; Joo and Zhao, 2017; Li et al., 2018; Zheng et al., 2018) and increasingly discharged into the environment (Gil-Allue et al., 2015; Park and Yeo, 2016; Peters et al., 2018;

\footnotetext{
* This paper has been recommended for acceptance by Baoshan Xing.

* Corresponding author. College of Environment, Zhejiang University of Technology, Hangzhou, 310032, PR China.

** Corresponding author.

E-mail addresses: zhqcai@cczu.edu.cn (Z. Cai), hfqian@zjut.edu.cn (H. Qian).
}

Qian et al., 2013; Shweta et al., 2016). Freshwater systems are one of the most important sinks of AgNPs, which raises concerns on $\mathrm{Ag}$ toxicity particularly to metal-sensitive microbial aquatic organisms (Metreveli et al., 2015; Schaumann et al., 2015). Measured (Peters et al., 2018; Zhang et al., 2019) or modeled (Batley et al., 2013) AgNPs concentrations are usually not high $\left(<1 \mu \mathrm{g} \mathrm{L}^{-1}\right)$ in natural surface water although a business-as-usual scenario will lead to an increase in AgNPs concentrations over time. It is of note, however, that contamination by AgNPs (and the associated dissolved Ag ions) has been observed in surface water near a model house treated with AgNPs-containing paints, with measured concentrations of total dissolved Ag reaching $145 \mu \mathrm{g} \mathrm{L}^{-1}$ following a rain event (Kaegi 
et al., 2010).

Dissolved Ag (released due to AgNPs dissolution) and AgNPs can be toxic to aquatic microorganisms (bacteria, fungi, and plankton) at trace concentrations (Reidy et al., 2013). Investigations of AgNPs toxicity in real or mimicked natural aquatic environments helped to gain knowledge on the toxicity of AgNPs to freshwater or seawater microbial communities. Previous studies indicated that a shortterm exposure to $10-20 \mu \mathrm{g} \mathrm{L}^{-1}$ AgNPs could temporarily affect bacterial community respiration and change the bacterial community composition (Das et al., 2012), and microorganisms tended to form microbial aggregates to protect themselves against AgNPs toxicity (Tang et al., 2018). AgNPs also had negative impacts on zooplankton grazing and the photosynthesis of phytoplankton at AgNPs concentrations $>500 \mu \mathrm{g} \mathrm{L}^{-1}$ (Baptista et al., 2015). Moreover, the microbial community structure and functional capacities of freshwater biofilms would also be changed by silver nanoparticles (Liu et al., 2018a; Barker et al., 2018).

Even though a few studies have revealed parts of the mechanisms of toxicity of AgNPs at the molecular level in aquatic environments (Boenigk et al., 2014; Zheng et al., 2017), the occurrence and causes of different toxic effects of AgNPs among bacterial and eukaryotic microorganisms and their transcriptional responses have to the best of our knowledge seldom been tested in natural systems. Laboratory studies indeed indicate that differences between toxicity patterns of AgNPs and detoxification mechanisms in bacterial and eukaryotic microorganisms exist (Chen et al., 2016; Qian et al., 2016). AgNPs were much more toxic to the growth, photosynthesis, antioxidant systems, and carbohydrate metabolism of the blue-green algae Microcystis aeruginosa than to the green alga Chlorella vulgaris grown in laboratory batch cultures (Chen et al., 2016). C. vulgaris could efficiently detoxify reactive oxygen species induced by AgNPs by enhancing antioxidant enzymes while $M$. aeruginosa failed to detoxify in this way under the same AgNPs concentration exposure (Qian et al., 2016).

The present study aims to determine the effects of AgNPs (initial total concentration $=10 \mu \mathrm{g} \mathrm{L}^{-1}$ ) on aquatic microbial communities in microcosms with emphasis on cyanobacteria and eukaryotic phytoplankton using state-of-the-art metatranscriptomic analyses. First, we analyzed the effects of AgNPs on the structure of aquatic microbial communities. Second, we investigated different responses of aquatic microbial eukaryota and bacteria after exposure to AgNPs. The present study brings new insights into the mechanisms of toxicity and the effects of a trace concentration of AgNPs on an aquatic microbial community.

\section{Material and methods}

\subsection{AgNPs characterization}

The citrate-coated AgNPs (mean diameter $=10 \mathrm{~nm}$, AGSWMB1000C) were purchased from Shanghai Huzheng Nano Technology Co. LTD (Shanghai, China) and suspended in Milli-Q water by sonication ( $20 \mathrm{kHz}$ and $100 \mathrm{~W}$ bath at $25^{\circ} \mathrm{C}$ ) for 30 min using an ultrasonic cleaner. The stability of AgNPs suspensions in medium $(1 \mathrm{mg} / \mathrm{L})$ was evaluated by transmission electron microscopy in our previous studies (Qian et al., 2016; Ke et al., 2018). The hydrodynamic size of AgNPs distribution between 63 and $67 \mathrm{~nm}$. Dissolved $\mathrm{Ag}$ was measured by ultrafiltration at $\mathrm{t}=0$ and $\mathrm{t}=96 \mathrm{~h}$ in the culture medium BG-11. It was found that the concentration of dissolved $\mathrm{Ag}$ (dissolution rate around 1.4\%) did not change much over 96 h (Qian et al., 2016).

\subsection{Microcosms and AgNPs treatments}

Freshwater samples were collected from the Mei-liang Bay in
Lake Taihu $\left(30^{\circ} 55^{\prime} 40^{\prime \prime}-31^{\circ} 32^{\prime} 58^{\prime \prime} \mathrm{N}\right.$; $119^{\circ} 52^{\prime} 32^{\prime \prime}-120^{\circ} 36^{\prime} 10^{\prime \prime}$ E, March 2017). The samples were filtered through a $0.22-\mu \mathrm{m}$ aperture pinhole filter membrane, and then the filter mass was resuspended in a modified BG-11 medium (chemical composition described in Table S1, and materials required for algal cultures were all pre-autoclaved or filter-sterilized before use) to prepare the microbial community stock culture (MTC). Please note that the lake water may contain traces of pollutants like pesticides. In order to solely study the influence of AgNPs on the plankton community in the microcosm and to eliminate the effects of other pollutants possibly present, we transferred the plankton community from the lake water to a pollution-free artificial medium. The MTC (including bacteria, fungi, algae, zooplankton and other microorganisms) was then inoculated into the modified BG-11 medium $(2 \mathrm{~L})$ contained in a beaker. The reason why the plankton community from the lake water was transferred to an artificial medium is that i) the lake water may contain traces of pollutants and a pollutant-free environment was needed. ii) adequate nutrients would promote the growth of microbes and more biomass would be available in the modified medium for RNA extraction. The optical density of all replicated microcosms at $680 \mathrm{~nm}$, a proxy for algal biomass, was adjusted to 0.01 , i.e., ca. $0.07 \mathrm{mg}$ Chlorophyll-a (Chl-a) $\mathrm{L}^{-1}$. After inoculation, AgNPs were added in the microcosms $(2 \mathrm{~L})$ at initial concentrations of $0.1,1,10,100$ and $1000 \mu \mathrm{g} \mathrm{L}^{-1}$ (each in 3 triplicates). This large range in AgNPs concentrations encompassed environmentally realistic concentrations as well as higher concentrations that are commonly used in short-term laboratory experiments (Liu et al., 2018b; Qian et al., 2016). The microcosms were placed in an artificial greenhouse at $25 \pm 0.5^{\circ} \mathrm{C}$ under cool-white fluorescent light $\left(46 \mu \mathrm{mol} \mathrm{m} \mathrm{m}^{-2} \mathrm{~s}^{-1}\right)$ with a $12 \mathrm{~h}: 12 \mathrm{~h}$ light: dark cycle and were agitated three times a day.

\subsection{Measurement of Chl-a and Ag in the microcosms}

Water samples in the control and in AgNPs-treated microcosms were collected to measure the Chl-a content with N, N-Dimethylformamide as described by Inskeep and Bloom (1985). The total dissolved Ag concentration was determined in the microcosms initially and after 7 days of exposure. Briefly, microcosm water samples $(10 \mathrm{~mL})$ were successively passed through a $0.45-\mu \mathrm{m}$ aperture pinhole filter, diluted in $\mathrm{HNO}_{3}$ (final concentration $=5 \% \mathrm{v}$ / $\mathrm{v} \mathrm{HNO}_{3}$ ) and the total Ag was determined using ICP-MS (NexION $300 \mathrm{X}$, PerkinElmer, USA). To measure the Ag concentrations in suspension (including elemental Ag in organisms and in the bulk solution), unfiltered microcosm samples were digested with $30 \%$ $\mathrm{H}_{2} \mathrm{O}_{2}(5 \mathrm{~mL})$ and $\mathrm{HNO}_{3}$ (final concentration $=5 \% \mathrm{v} / \mathrm{v} \mathrm{HNO}_{3}$ ) and then successively passed through a $0.45-\mu \mathrm{m}$ aperture pinhole filter. Ag content was determined with ICP-MS (Luo et al., 2018). All experiments were repeated three times independently.

\subsection{Metatranscriptomic sample preparation and sequencing}

Three control microcosms (Con1, Con2, and Con3) as well as three microcosms containing $10 \mu \mathrm{g} \mathrm{L}^{-1}$ AgNPs (initial concentration) (AgNPs1, AgNPs2, AgNPs3) were harvested after seven days of culture for silver measurements and metatranscriptomic analyses. After 7 days of culturing, water samples from the six aquatic microcosms were centrifuged at $6500 \mathrm{~g}$ for $5 \mathrm{~min}$ and the supernatant was filtered through a $0.2-\mu \mathrm{m}$ filter membrane. Microorganisms RNA was extracted from the filters using TRIzol Reagent (Invitrogen, Thermo fisher, USA) and the RNeasy Mini Kit (Qiagen, Germany). RNA was quantified and qualified with an Agilent 2100 Bioanalyzer (Agilent Technologies, Palo Alto, CA, USA), and a NanoDrop (Thermo Fisher Scientific Inc.). RNA integrity was evaluated using gel electrophoresis (1\% agarose formaldehyde). One microgram 
total RNA with a RIN (RNA Integrity Number) value above seven was used for library preparation. The rRNA was depleted from total RNA using the Ribo-Zero rRNA Removal Kit (Bacteria) (Illumina). The sequences were processed and analyzed by GENEWIZ (Suzhou, China). More detailed information on the library preparation and sequencing was as previously described (Lu et al., 2019a). The raw data have been submitted to the NCBI Sequence Read Archive (SRA) database with accession numbers SAMN10362734 to SAMN10362739.

\subsection{Analysis of differentially expressed genes}

In total, 807.1 $\mathrm{M}$ clean reads were generated after filtering lowquality reads and trimming adaptor sequences from the raw reads. The processed clean reads were assembled and annotated functionally and taxonomically. Taxonomies were annotated by using the NCBI Non-Redundant Protein (Nr) database. In-house scripts were used to find genes associated with significant differential expression in KEGG (Kyoto Encyclopedia of Genes and Genomes) pathways. We used FPKM (expected number of Fragments Per Kilobase of transcript sequence per Millions base pairs sequenced) to calculate the relative transcript level of genes. More detailed information on the data analysis and annotation process was described by Lu et al. (2019b).

\subsection{Statistical analysis}

All experiments were repeated three times independently. Data are presented as mean \pm standard error. Significant differences among treatments (Chl-a content and $\mathrm{Ag}$ concentrations in the microcosm) were tested with one-way ANOVAs followed with Fisher's PLSD test (StatView 5.0). Differences were considered statistically significant when $p<0.05$. In meta-transcriptomic analysis, gene expression folds $\geq 2$ and FDR (false discovery rate) $\leq 0.05$ were set as thresholds to determine if genes were significantly differentially expressed.

\section{Results and discussion}

\subsection{Chl-a and total Ag concentrations in the microcosm}

The Chl-a content in the microcosms, which was used as a proxy for algal cell density, was influenced by the AgNPs treatments
(Fig. 1a). The two lowest AgNPs concentrations (0.1 and $1 \mu \mathrm{g} \mathrm{L}^{-1}$ ) had no significant inhibitory effects on Chl-a concentrations, suggesting that the algal communities were able to tolerate the presence of these trace concentrations of AgNPs. However, in the microcosms treated with 10 and $100 \mu \mathrm{g} \mathrm{L}{ }^{-1}$, Chl-a concentrations were slightly reduced over the first $5 \mathrm{~d}$ of exposure and significantly inhibited after $7 \mathrm{~d}$ of exposure. The treatment with an initial concentration of $1000 \mu \mathrm{g} \mathrm{L}{ }^{-1}$ AgNPs most strongly decreased the Chl-a concentration during the 7-d experiments. For further in-depth metatranscriptomic experiments, a concentration of $10 \mu \mathrm{g} \mathrm{L}^{-1}$ initial AgNPs was selected as this was the lowest tested concentrations significantly affecting the Chl-a concentration (by 24\%) over the 7-day microcosm experiment.

Regarding the release of $\mathrm{Ag}^{+}$ions, we have previously detected that the concentration of dissolved $\mathrm{Ag}$ was only $1.4 \%$ (96 h) in BG11 medium (Qian et al., 2016), possibly because $\mathrm{Cl}^{-}$ions as well as other anions present like $\mathrm{CO}_{3}^{2-}$ may quickly bind to the released $\mathrm{Ag}^{+}$ ions, cover the surface of the NPs, and prevent further ion release (Lin et al., 2015). It is thus unlikely that dissolved Ag ion play a major role in inducing toxicity of AgNPs to the microbial community. As shown in Fig. 1b, the measured Ag concentration at the start of the experiment in the filtered microcosm was $7.48 \mu \mathrm{g} \mathrm{L}^{-1}$, which was far less than the amount of Ag particles added $\left(10 \mu \mathrm{g} \mathrm{L}^{-1}\right)$. This finding indicated that the AgNPs tended to be lost rapidly from the unfiltered water, likely by aggregation and sedimentation, and as well as adsorption on and absorption in microorganisms (Jiang et al., 2017). The total Ag concentrations in the filtered microcosm water decreased from $7.48 \mu \mathrm{g} \mathrm{L}^{-1}$ to $0.23 \mu \mathrm{g} \mathrm{L}^{-1}$ after the 7-day exposure period (Fig. 1b), which indicates that most Ag (including AgNPs and dissolved ionic Ag) was lost during the 7-day exposure. Therefore, the peak concentration of AgNPs in natural water systems is expected to be much higher than the detected dissolved concentrations in lakes, streams or other surface waters. After 7 days, the total Ag concentration measured in the unfiltered water was $2.78 \mu \mathrm{g} \mathrm{L}^{-1}$, and therefore about $2.55 \mu \mathrm{g} \mathrm{L}^{-1} \mathrm{Ag}$ (concentration in the unfiltered water minus the concentration in the filtered water) had accumulated in plankton or had aggregated over the 7$\mathrm{d}$ culture. The strong loss of $\mathrm{Ag}$ over time in the microcosms is consistent with the results of Jiang et al. (2017), who found that the sediment surface layer was the main sink of $\mathrm{Ag}$ originating from AgNPs. a

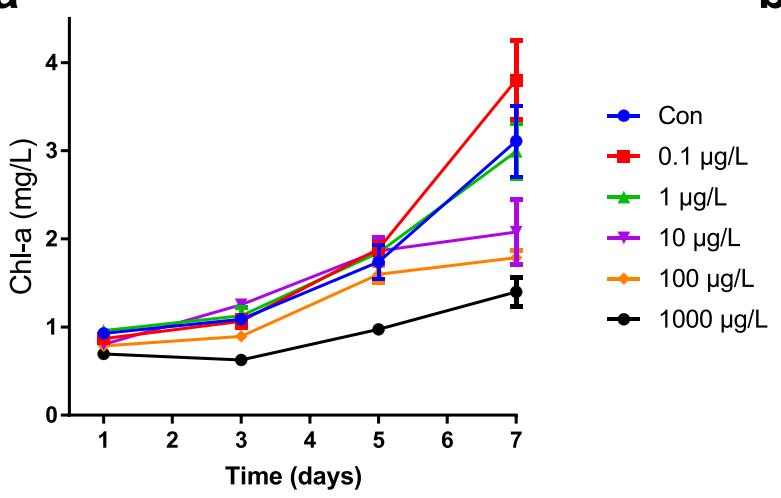

b

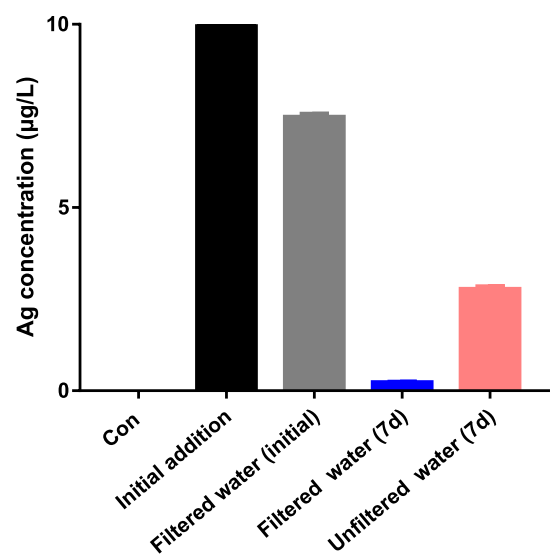

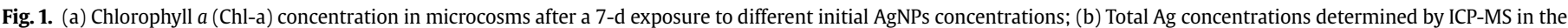

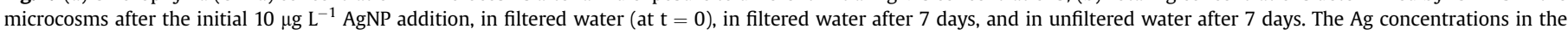
control (BG-11 medium) were below the detection limit. 


\subsection{Transcriptome sequencing of the microcosms}

The global impact of AgNPs on the function and taxonomy of photosynthetic and heterotrophic microbiota at the molecular level was investigated using metatranscriptomic analysis after the 7-day exposure. The total number of raw reads from the six samples (Con1, Con2, Con3, AgNPs1, AgNPs2, AgNPs3) were 826.3 million (M). After assembly of clean reads, $1.15 \mathrm{M}$ unigenes were obtained and $37.6 \%$ of them $(0.43 \mathrm{M})$ could be annotated by the KEGG database. The expected number of fragments per kilobase of transcript sequence per millions base pairs sequence (FPKM) was used to calculate relative transcript level of genes. Detailed statistics of clean data, sample transcript and FPKM interval from the six microcosms are shown in Dataset 1. Up-regulated and downregulated genes are represented with a volcano plot (Fig. S1), which shows fold change (AgNPs/Con) and FDR (false discovery rate) values. Compared to the control group, RNA-Sequencing analyses identified 184,962 genes whose expression changed significantly (foldchange $>2$, FDR $<0.05$ ) after treatment with $10 \mu \mathrm{g} \mathrm{L}^{-1}$ AgNPs for $7 \mathrm{~d}$, whereas 89,344 of these genes were upregulated and 95,618 were downregulated, as visualized with a volcano plot (Fig. S1). This large amount of differentially expressed genes suggests that the AgNPs treatment $\left(10 \mu \mathrm{g} \mathrm{L}^{-1}\right)$ impacted a variety of molecular pathways of the microbial community in the microcosm.

\subsection{AgNPs are much more toxic to bacteria than to eukaryota}

Taxonomic proportions were calculated based on the relative abundance of taxonomically annotated transcripts (RAT) of six microcosms at different levels, and the results are shown in Dataset 2. The RAT value does not represent microbial biomass, but rather the changes in transcriptional activity among species, which represents the active metabolic states and function of the microbial community. In the control microcosms, $24.4 \%$ of the taxonomically annotated sequences were from bacteria and this sharply decreased to $5.3 \%$ in the AgNPs group (Fig. 2a), implicating that the aquatic bacteria were negatively affected due to the antimicrobial properties of AgNPs (Maurer-Jones et al., 2013; Zhang et al., 2016). A principal component analysis (PCA) (PERMANOVA, $\mathrm{R}^{2}=0.90$, $\mathrm{P}=0.11$ ) showed that the community structure (including both bacteria and eukaryota) in the AgNPs group was different from the community structure in the control (Fig. 2b). The relative proportion of microorganisms (at the phylum level) using FPKM values of the six microcosms after the 7-d exposure is shown in Fig. 2c. The RAT of Cyanobacteria dramatically decreased by $90 \%$ in the AgNPs group compared to the control, while the RAT of Chlorophyta increased by $\sim 32 \%$, suggesting that AgNPs were much more toxic to Cyanobacteria than to Chlorophyta (if any toxicity of AgNPs occurred to the Chlorophyta in the microcosms) and that Chlorophyta outcompete Cyanobacteria. In the six microcosms, Monoraphidium and Synechococcus were the major genera in Chlorophyta and Cyanobacteria, respectively. AgNPs toxicity bioassays in laboratory batch cultures of Monoraphidium sp. and Synechococcus sp. (Fig. S2) showed that $10 \mu \mathrm{g} \mathrm{L}^{-1}$ AgNPs reduced the growth of Monoraphidium sp. by $50 \%$ compared to the control. Synechococcus sp. almost stopped growing at AgNPs concentrations below $2 \mu \mathrm{g} \mathrm{L}^{-1}$, indicating that the two algae in monoculture were both suppressed by AgNPs, with Synechococcus sp. being much more sensitive than Monoraphidium sp. Interestingly, the growth of Monoraphidium sp. was severely inhibited by the presence of $10 \mu \mathrm{g} \mathrm{L}^{-1}$ initial AgNPs in a laboratory batch monoculture whereas in the microcosms, this species increased in abundance. This difference in response to exposure to Ag of Monoraphidium sp. might be due at least partly to the reciprocal action with other microbes in the microcosms than in the laboratory batch cultures.

Besides Cyanobacteria, the RAT of most other bacteria, including Proteobacteria, Bacteroidetes, Firmicutes, Verrucomicrobia and Thermotogae, decreased significantly (by 72-99\%) after $7 \mathrm{~d}$ of exposure to $10 \mu \mathrm{g} \mathrm{L} \mathrm{L}^{-1}$ AgNPs. Interestingly, the RAT of Planctomycetes increased dramatically $(+136 \%)$ in response to AgNPs exposure, indicating AgNPs resistance in bacteria of this phylum.

The RAT of heterotrophic eukaryota including fungi and a
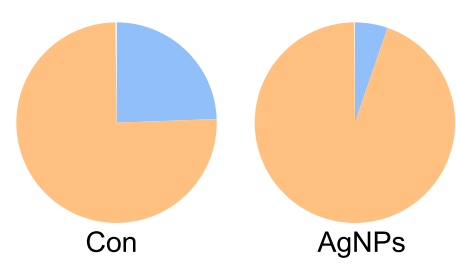

b

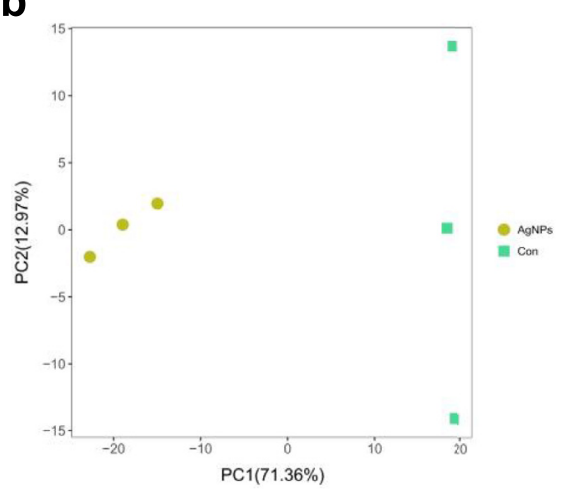

C

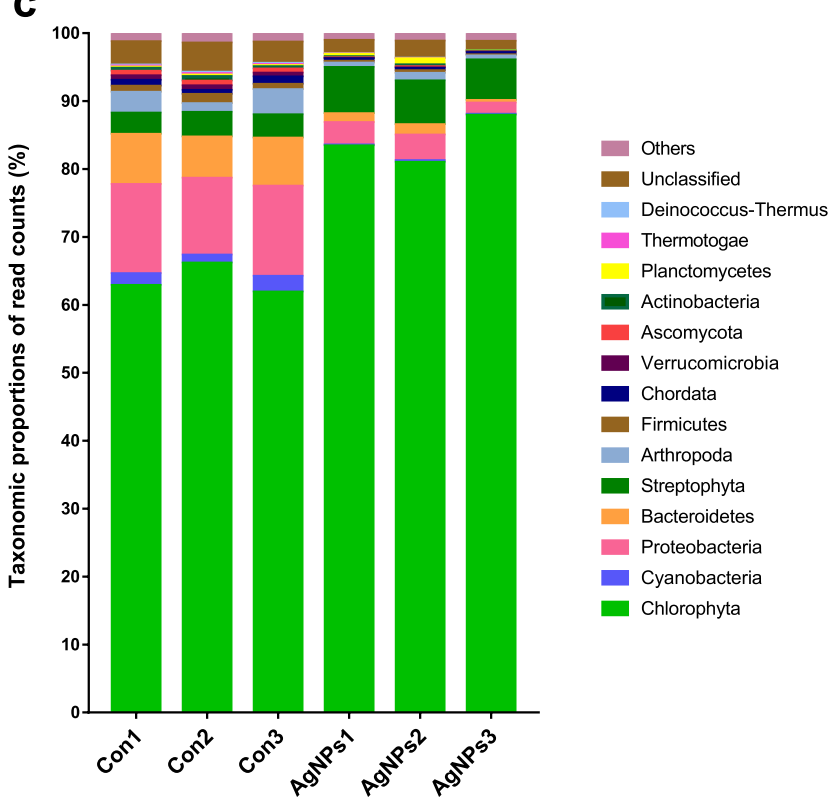

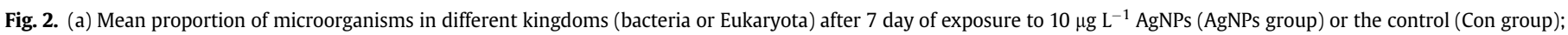

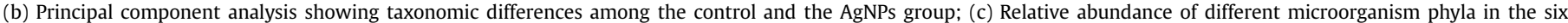

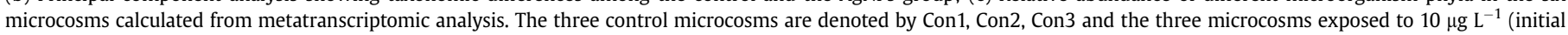
added concentration) AgNPs for 7 days are denoted by AgNPs1, AgNPs2, AgNPs3. 
zooplankton decreased after $7 \mathrm{~d}$ of exposure to $10 \mu \mathrm{g} \mathrm{L}^{-1}$ AgNPs compared to the abundance in the control. For instance, the RAT of Ascomycota and Arthropoda decreased by $79 \%$ and 71\%, respectively, compared to the control. The reason is possibly the reduction of the amount of food available.

\subsection{Global functional changes in microcosms in response to AgNPs exposure}

Analysis of the abundance of KEGG orthologs (KOs) can identify the key functional features of the microbiota in microcosms (Lu et al., 2018). In total, the $0.43 \mathrm{M}$ annotated unigenes were organized into 11,911 different KOs (Dataset 3), which can be further organized into 307 small metabolic pathways at the KEGG level 3 (Figs. 4 and 5, Dataset 4 and 5) and 44 metabolic subsystems at the KEGG level 2 (Fig. 3). Comparing the transcripts, there was a significant difference between the control and AgNPs treated groups in the relative abundance of the observed functional assignments $(p<0.05)$. These subsystems belong to six basic metabolic systems at KEGG level 1. Fig. 3 shows the effect of AgNPs on aquatic microbial functions as determined with 21 subsystems belonging to four metabolic systems (i.e., metabolism, genetic information processing, environmental information processing, and cellular processes). Sixteen of the 21 subsystems were significantly over- or under-expressed, but most subsystems (11) were underexpressed in response to AgNPs (Fig. 3). For instance, the most highly over-expressed KEGG level 2 categories in the AgNPs-treated group relative to the control were translation $(+78 \%$ compared to control), transcription $(+36 \%)$ and energy metabolism $(+16 \%)$. Gene transcripts involved in Energy metabolism (i.e., mainly in photosynthesis) were abundant in both the AgNPs-treated group (49.7\% of the total transcripts per sample) and the control (42.8\%). This could be explained by the growth advantage of photosynthetic eukaryota in the control group and this advantage was further strengthened after AgNPs exposure (Fig. 2c). The most underexpressed subsystems were related to replication and repair ($77 \%$ compared to control), metabolism of other amino acids (- 76\%) and membrane transport (-72\%). Taken together, the observed variation in transcript abundance among subsystems in the microcosm highlights that the metabolism of the planktonic community was profoundly affected in response to the AgNPs treatment.

\subsection{Metabolic pathways in eukaryota and bacteria in response to AgNPs exposure}

The global functional variation (KEGG level 2) in Fig. 3 suggests that the AgNPs treatment significantly changed the function of the

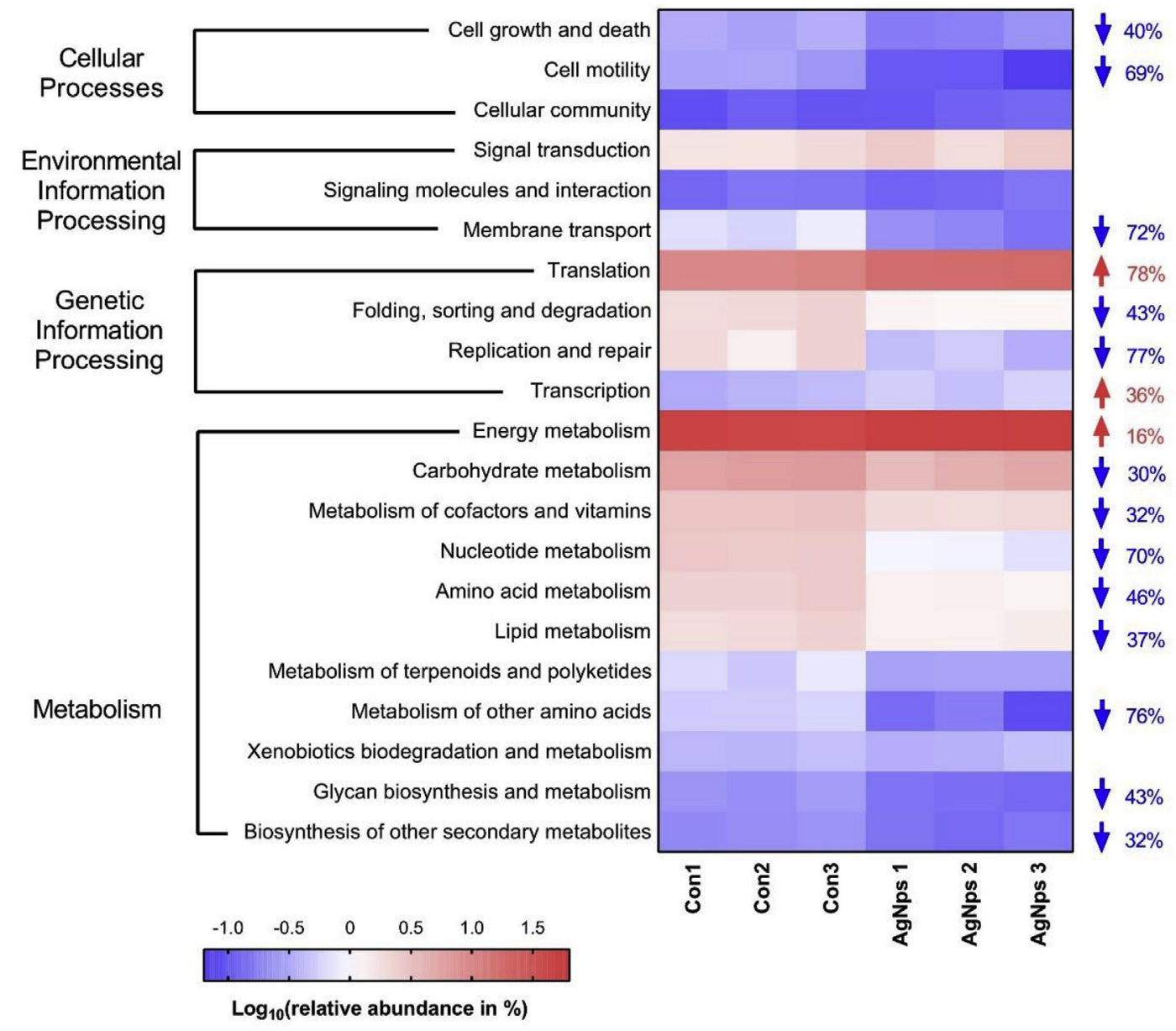

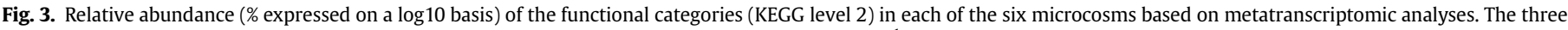

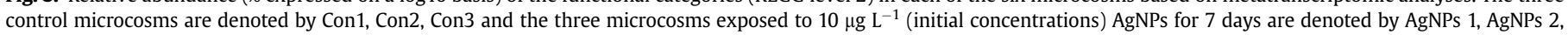

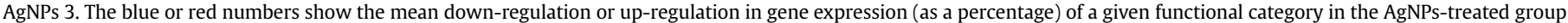

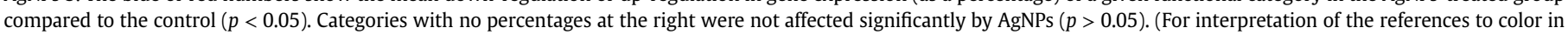
this figure legend, the reader is referred to the Web version of this article.) 
microbial community. However, since the growth states of aquatic eukaryota and bacteria responded quite differently to AgNPs, indepth transcript analysis of the microbial community was needed to further understand the different effects of AgNPs on eukaryota and bacteria. The sequences of eukaryota and bacteria were thereby distinguished and dissected at KEGG level 3 (Dataset 4) and level 4 (Dataset 5). Pathways (belonging to four metabolic systems mentioned in the previous section) were selected as over- or underexpressed in the AgNPs-treated microcosms according to their transcripts' fold change and relative abundance (indicated by FPKM) compared with the control group, as shown in Fig. 4 (for eukaryota) and in Fig. 5 (for bacteria). The global effects of AgNPs on 6 categories of the metabolism of eukaryotic and bacterial organisms are detailed below.

In eukaryota, the relative abundance of transcripts belonging to 128 out of 306 functional categories (41.8\%) at KEGG level 3 were observed to be significantly different between the control and the AgNPs-treated microcosms (Dataset 4). This relatively high proportion was surprising with regards to the resistance of photosynthetic eukaryota to AgNPs (Figs. 1, 2a and c) although heterotrophic eukaryota were sensitive to AgNPs (Fig. 2c).

Overall, the bacterial community structure and the relative abundance of bacterial metabolic pathways changed more dramatically than those of eukaryota as expected because of the antibacterial properties of AgNPs. Pathways closely related to cyanobacteria were significantly under-expressed in the AgNP-treated group (Fig. 5, dataset 4); including carotenoid biosynthesis (- $91 \%$ compared with control), photosynthesis-antenna proteins (-97\%) and photosynthesis (- 86\%). Furthermore, pathways related to vitamin metabolism, translation and other important metabolic processes were also greatly differentially expressed in response to AgNPs (Fig. 5, Dataset 4). The detailed AgNP transcriptional responses in bacteria and eukaryota are discussed in the 6 sections below.

\section{Energy metabolism}

Photosynthesis is the pathway for energy production in the light in eukaryotic phytoplankton and cyanobacteria (Nelson and BenShem, 2004). In eukaryota, the relative expression level of genes related to photosynthesis increased slightly in the AgNPs-treated microcosms compared to the control (Fig. 4), which is in line with the observed general increase in the relative abundance of photosynthetic eukaryotic organisms over heterotrophic organism. A set of genes involved in the assembly of photosystem II (PSII), such as $p s b \mathrm{~F}(+740 \%), p s b \mathrm{R}(+597 \%)$ and $p s b Z(+388 \%)$, were significantly over-expressed, while genes involved in the photosystem I (PSI) assembly did not change much. The rise in the transcription of genes related to the PSII assembly under the AgNPs treatment could be related to the rise in the RAT of eukaryotic algae, and we postulate that up-regulation at the level of the PSII may be triggered in response to the presence of AgNPs. However, oxidative phosphorylation, which is the cornerstone of aerobic cellular metabolism (Meyer et al., 2009), was significantly supressed (dataset 4), indicating that energy generation through oxidative metabolism was negatively affected by AgNPs. This is consistent with the decrease in the RAT of heterotrophic eukaryota (Fig. 3c) in

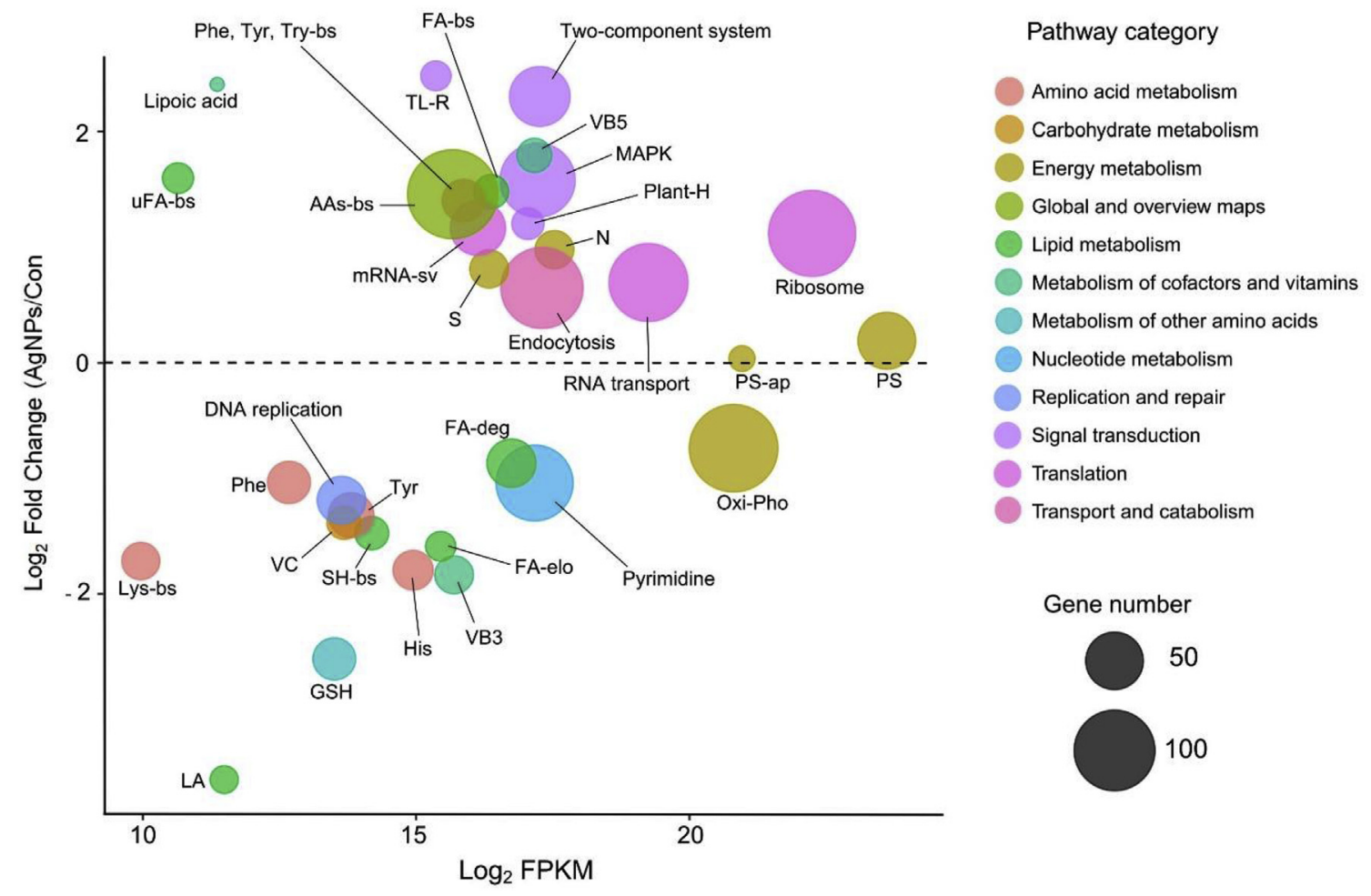

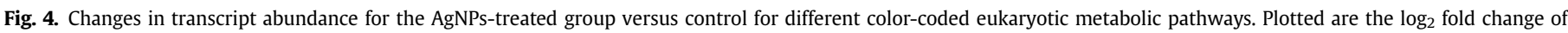

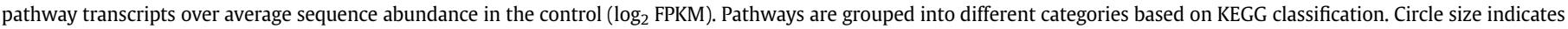

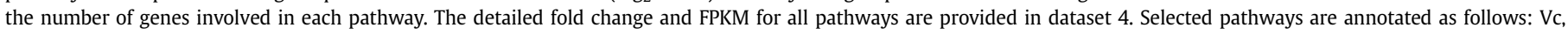

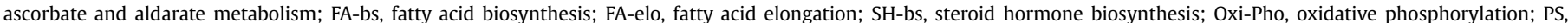

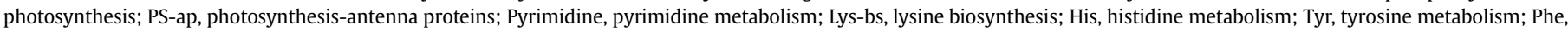

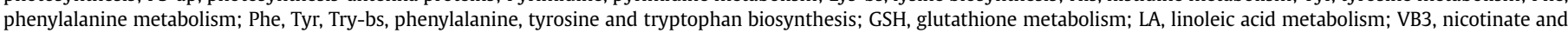

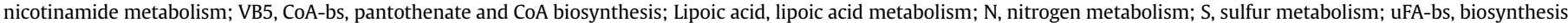

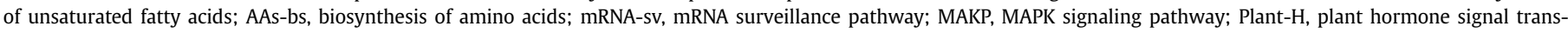
duction; TL-R, Toll-like receptor signaling pathway. 


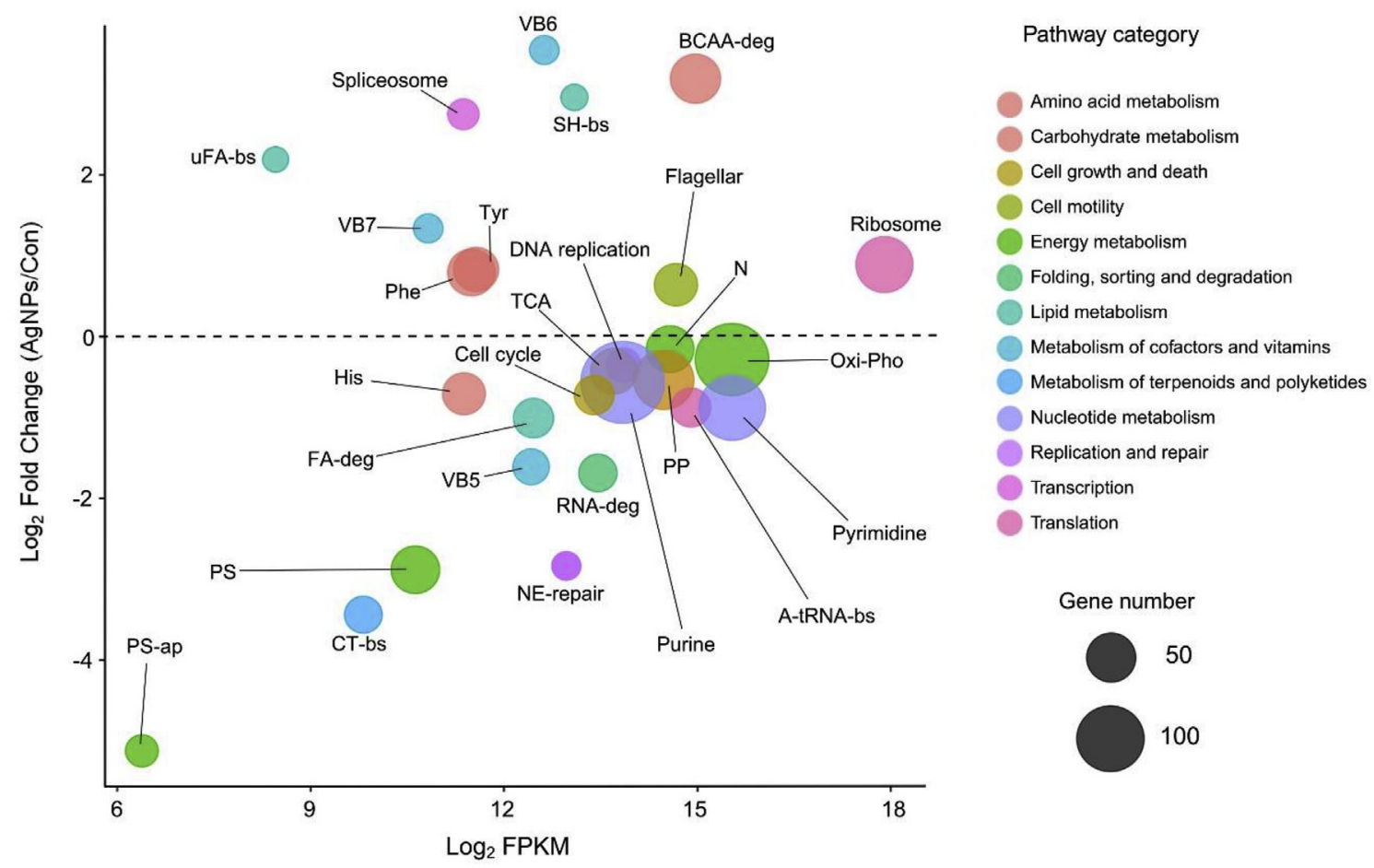

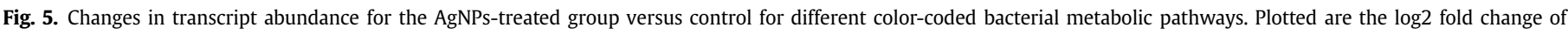

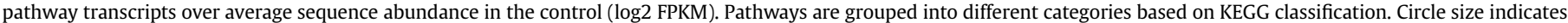

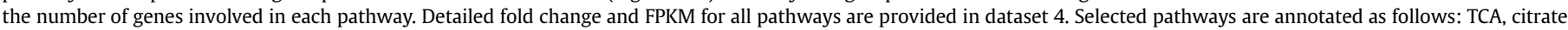

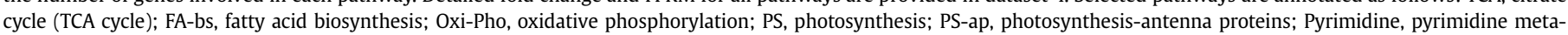

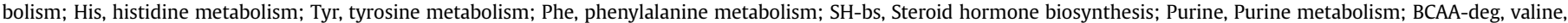

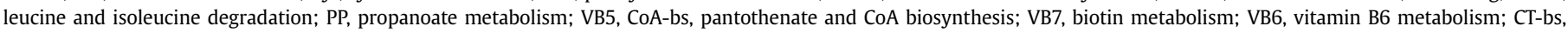

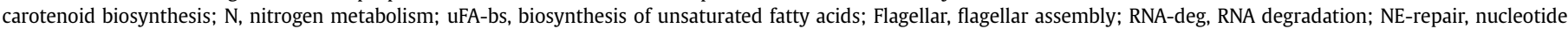
excision repair.

response to the AgNPs stress. The transcription of genes related to sulfur metabolism increased in response to AgNPs treatment, including sulfate transporters (cysP, cysU, cys $A, c y s W$ ), sulfite reductase (cysI, cysJ) and cysteine synthase (cysM, cysK), most of which were related to cysteine metabolism (Mansilla et al., 2000). Cysteine is a strong $\mathrm{Ag}^{+}$-binding ligand that can abolish the inhibitory effects caused by $\mathrm{Ag}^{+}$(Chen et al., 2018). Enhanced cystine-related metabolism in eukaryotic organisms might therefore play a role in alleviating the toxicity of $\mathrm{Ag}^{+}$released by AgNPs. The transcription of genes involved in nitrogen metabolism increased in response to AgNPs exposure, including nitrite reductase $(n i r D)$ and glutamate synthase and dehydrogenase $(g l t D, g d h A)$. In higher plants, genes related to nitrogen metabolism are often enhanced to resist various environmental stressors (Xun et al., 2017; Zhu et al., 2018), suggesting that eukaryota in the microcosm, which were mainly composed of Chlorophyta, may used a similar strategy to cope with stressful conditions as reported for higher plants. By contrast, in bacteria, photosynthesis related pathways were strongly inhibited, especially for the pathway "photosynthesis - antenna proteins" (- 97\%), which can absorb and transmit light energy to the photosynthetic reaction center. This indicates that photosynthesis might be an important target of AgNPs. Meanwhile, other energy metabolism pathways including oxidative phosphorylation did not decrease sharply, indicating that heterotrophic bacteria could still maintain their aerobic metabolism.

\section{Ribosome}

In eukaryota, transcripts of 108 out of 132 ribosome related genes were significantly over-expressed in response to the AgNPs treatment. By contrast, in bacteria, most ribosome related genes were under-expressed. Interestingly, there was a remarkable gene (K02946, RP-S10, encoding for the small subunit ribosomal protein S10) that was acutely over-expressed (for $\sim 20$-fold) and its transcripts were accounting for $75 \%$ of the whole ribosome pathway in the AgNPs group. In bacteria, there also was a special gene in the spliceosome pathway named RBMX (K12885, encoding for heterogeneous nuclear ribonucleoprotein G), which was also dramatically over-expressed (for $\sim 86$-fold) after AgNPs treatment and its transcripts accounted for $\sim 84 \%$ of the whole ribosome pathway in the AgNPs group. In previous studies (Fan et al., 2018; Xie et al., 2015), plants or eukaryotic algae also showed upregulation of ribosome-related genes under environmental stress like AgNPs treatment, which is in according with the findings of the present study. Generally, the results suggest that eukaryota may be particularly effective to strengthen transcription processes and repair in response to cell damages due to the presence of AgNPs compared to bacteria, although a few exceptions exist in particular ribosomal bacterial proteins.

\section{Vitamin metabolism}

Phytoplankton requires multiple B vitamins as growth factors, such as vitamin $B_{12}$ (cobalamin), vitamin $B_{6}$, biotin, pantothenate and lipoic acid (Croft et al., 2006; Gong et al., 2017). In eukaryota, transcripts of pathways related to the synthesis of pantothenate $(+249 \%)$ and lipoic acid $(+334 \%)$ were significantly increased, while that of the pathway of nicotinate and nicotinamide metabolism were under-expressed (-72\%) (Fig. 4, Dataset 4$)$. In bacteria, pathways related to vitamin $\mathrm{B}_{6}(+1062 \%)$ and biotin $(+152 \%)$ were over-expressed but the pathway of pantothenate and CoA 
biosynthesis were under-expressed (-67\%) (Fig. 4, Dataset 4). These results indicate that eukaryota and bacteria are impacted differently at the vitamin level by AgNPs. Interestingly, in bacteria, the relative abundance of four genes involved in cobalamin biosynthesis (i.e., cobW or K02234: cobalamin biosynthesis protein CobW, cobC or K02225: cobalamin biosynthesis protein CobC) increased by $32 \%$ and $444 \%$, respectively, in the AgNPs -treated microcosms relative to the control (See Dataset 5). Meanwhile, two cobalamin transporter genes in the pathway $A B C$ transporters (i.e., btuB or K16092: vitamin $B_{12}$ transporter, btuF or K06858: vitamin $B_{12}$ transport system substrate-binding protein) also increased by 85\% and 522\%, respectively, after AgNPs exposure. Since several eukaryotic species (including fungi and many Chlorophytes) cannot synthesize cobalamin de novo and rely on mutualistic bacteria to acquire this vitamin (Croft et al., 2005; Graham et al., 2004), our results indicate that AgNPs stimulated rather than decreased cobalamin supply to eukaryotic organisms through mutualistic interactions even though bacteria metabolism was strongly negatively affected by AgNPs.

\section{Amino acids and fatty acids metabolism}

In eukaryota, the relative transcription levels of most amino acids related pathways were not largely affected. However, pathways related to phenylalanine, tyrosine and tryptophan biosynthesis were significantly over-expressed $(+166-177 \%)$ and four metabolic pathways (i.e., phenylalanine metabolism, tyrosine metabolism, lysine biosynthesis and histidine metabolism) were significantly under-expressed (- 51-71\%) by the AgNPs exposure compared to the control. To sum up, it seems that the eukaryota tend to accumulate aromatic amino acids (AAA) under AgNPs exposure. In bacteria, histidine metabolism was also underexpressed (-39\%) in the AgNPs group compared to the control, while the phenylalanine and tyrosine metabolism were significantly over-expressed ( $+73 \%$ and $+77 \%$, respectively), and it was noteworthy that the branched chain amino acids (BCAA) -related pathway "Valine leucine and isoleucine degradation" was overexpressed by 808\%. This was mostly linked to modulation of the transcription of the gene acsA (K01907, encoding for acetoacetylCoA synthetase, AACS) whose relevant transcription increased by $1813 \%$ in response to AgNPs exposure. AACS is an enzyme responsible for cholesterol and fatty acid synthesis in the cytosol (Hasegawa et al., 2018), and its higher expression level may help cells synthesize cholesterols that can then be used to support growth when environmental conditions become more favorable (Gong et al., 2017). The results above suggest that, following exposure to AgNPs, eukaryota tend to accumulate AAA in response to the presence of AgNPs, while the bacteria consume extra BCAA, the insufficiency of which negatively affects the basic cell metabolism. The AgNPs can easily cause cell membrane damage (Reidy et al., 2013; Zheng et al., 2018), and more fatty acids, especially unsaturated fatty acids, were needed to repair the membrane structure (Barati et al., 2019). Therefore, it can be understood that the pathway "biosynthesis of unsaturated fatty acids" was overexpressed in both eukaryota (+205\%) and bacteria (+354\%) after AgNPs exposure.

\section{DNA replication and genetic repair}

Silver ions can impair DNA replication by uncoupling respiratory electron transport from oxidative phosphorylation and then inhibit respiratory chain enzymes (Duran et al., 2010). In both eukaryota and bacteria, the relative transcription level of DNA replication was decreased (dataset 4, dataset 5) after AgNPs exposure. It is also remarkable that the nucleotide excision repair pathway in bacteria was dramatically under-expressed (- 86\%), especially for the genes involved in excinuclease ABC and DNA helicase II (uvrA, uvrD and $u v r B$ ), the transcripts of which all decreased by $~ 90 \%$. Nucleotide excision repair is a mechanism to recognize and repair bulky DNA damage caused by compounds as well as other environmental stress (de Laat et al., 1999). The vulnerability in DNA replication and genetic repair induced a greater disadvantage to bacteria as compared to eukaryota.

\section{Signal transduction}

Signal transduction related pathways responded intensely after AgNPs treatment in both eukaryota and bacteria. The MAPK (mitogen-activated protein kinase) signaling pathway in eukaryota increased by $\sim 3$-fold relative to control. The MAPK cascade is a highly conserved module that is involved in various cellular functions, including cell proliferation, differentiation and migration (Chen et al., 2001), and its over-expression may generate specific kinases, such as the c-Jun N-terminal kinase (Harper and LoGrasso, 2001), to activate cellular alarm systems. In bacteria, the TGF-beta signaling pathway (ko04350) increased by 256-fold and the Rap1 signaling pathway (ko04015) increased by 232-fold in response to AgNPs treatment. Most of the over-expression in the two pathways, was due to two differentially-expressed genes: LTBP1 (K19559, latent transforming growth factor beta binding protein 1) and TEK (K05121, endothelial-specific receptor tyrosine kinase). Interestingly, to the best of our knowledge, both signaling pathways (i.e., TGF-beta and Rap1) were mentioned only in eukaryotic organisms, but not in bacteria: Rap1 might function in diverse processes, ranging from modulation of growth and differentiation to secretion, integrin-mediated cell adhesion and morphogenesis (Bos et al., 2001), while TGF-beta family members are structurally related secreted cytokines found in species ranging from worms and insects to mammals (Shi and Massague, 2003). The dramatic over-expression ( $>200$ folds) of the two pathways makes us believe that they may play roles in AgNPs detoxification in both bacteria and eukaryota.

\section{Conclusions}

The present study brings new insights into the transcriptional responses of AgNPs in natural freshwater microbial communities in microcosms. We show that AgNPs $\left(10 \mu \mathrm{g} \mathrm{L}^{-1}\right)$ in microcosms strongly affect the microbial community structure. Photosynthetic eukaryota were much more tolerant to AgNPs than cyanobacteria, and therefore dominate over time in the AgNPs treated microcosms. Metatranscriptomic analyses show that aquatic microbial eukaryota and bacteria respond quite differently to the AgNPs stress; eukaryota show a number of potential AgNPs detoxification mechanisms while bacteria show several signs of AgNPs toxicity with little signs of detoxification. Eukaryota could enhance nitrogen and sulfur metabolism, over-express translation-related genes, promote biosynthesis and decrease degradation of some amino acids, strengthen bacterial-algae mutualistic interactions and activate the MAPK signaling pathway to better tolerate the presence of AgNPs. By contrast, in bacteria, many pathways related to energy metabolism, DNA replication and genetic repair were seriously inhibited by AgNPs. The limited signs of AgNPs potential detoxification in bacteria included consumption of amino acid and activation of some stress signaling pathways. The current study unravels several cellular transcriptional responses explaining the differential toxicity of AgNPs among bacteria and eukaryota in the field. It provides a conceptual framework explaining at the molecular level why AgNPs could affect the structure of a lake microbial community, favoring eukaryotic algae rather than cyanobacteria. This interaction at the community level may lead to an ecological unbalance and needs to be noted in AgNPs risk assessment in freshwater systems. 


\section{Declaration of competing interest}

The authors declare that they have no competing interests.

\section{CRediT authorship contribution statement}

Tao Lu: Formal analysis, Writing - original draft. Qian Qu: Formal analysis. Michel Lavoie: Writing - original draft. Xiangjie Pan: Formal analysis. W.J.G.M. Peijnenburg: Writing - original draft. Zhigao Zhou: Formal analysis. Xiangliang Pan: Writing original draft. Zhiqiang Cai: Methodology, Writing - original draft. Haifeng Qian: Methodology, Writing - original draft.

\section{Acknowledgments}

This work was financially supported by the National Natural Science Foundation of China (21777144, 41907210, 21976161).

\section{Appendix A. Supplementary data}

Supplementary data to this article can be found online at https://doi.org/10.1016/j.envpol.2019.113727.

\section{References}

Baptista, M.S., Miller, R.J., Halewood, E.R., Hanna, S.K., Almeida, C.M.R. Vasconcelos, V.M., Keller, A.A., Lenihan, H.S., 2015. Impacts of silver nanoparticles on a natural estuarine plankton community. Environ. Sci. Technol. 49 (21), 12968-12974.

Barati, B., Gan, S.Y., Lim, P.E., Beardall, J., Phang, S.M., 2019. Green algal molecular responses to temperature stress. Acta Physiol. Plant. 41, 26.

Barker, L.K., Giska, J.R., Radniecki, T.S, Semprini, L, 2018. Effects of short- and longterm exposure of silver nanoparticles and silver ions to Nitrosomonas europaed biofilms and planktonic cells. Chemosphere 206, 606-614.

Batley, G.E., Kirby, J.K., McLaughlin, M.J., 2013. Fate and risks of nanomaterials in aquatic and terrestrial environments. Acc. Chem. Res. 46 (3), 854-862.

Boenigk, J., Beisser, D., Zimmermann, S., Bock, C., Jakobi, J., Grabner, D. Grossmann, L., Rahmann, S., Barcikowski, S., Sures, B., 2014. Effects of silve nitrate and silver nanoparticles on a planktonic community: general trends after short-term exposure. PLoS One 9 (4), e95340.

Bos, J.L., de Rooij, J., Reedquist, K.A., 2001. Rap1 signalling: adhering to new models. Nat. Rev. Mol. Cell Biol. 2 (5), 369-377.

Chen, S., Jin, Y.J., Lavoie, M., Lu, H.P., Zhu, K., Fu, Z.W., Oian, H.F., 2016. A new extracellular von Willebrand A domain-containing protein is involved in silver uptake in Microcystis aeruginosa exposed to silver nanoparticles. Appl. Microbiol. Biotechnol. 100 (20), 8955-8963.

Chen, Z., Gibson, T.B., Robinson, F., Silvestro, L., Pearson, G., Xu, B.E., Wright, A Vanderbilt, C., Cobb, M.H., 2001. MAP kinases. Chem. Rev. 101 (8), 2449-2476.

Chen, Z., Sheng, X., Wang, J., Wen, Y., 2018. Silver nanoparticles or free silver ions work? An enantioselective phytotoxicity study with a chiral tool. Sci. Total Environ. 610-611, 77-83.

Croft, M.T., Lawrence, A.D., Raux-Deery, E., Warren, M.J., Smith, A.G., 2005. Algae acquire vitamin B-12 through a symbiotic relationship with bacteria. Nature 438 (7064), 90-93.

Croft, M.T., Warren, M.J., Smith, A.G., 2006. Algae need their vitamins. Eukaryot. Cell $5(8), 1175-1183$

Das, P. Williams, C.J., Fulthorpe, R.R., Hoque, M.E., Metcalfe, C.D., Xenopoulos, M.A. 2012. Changes in bacterial community structure after exposure to silve nanoparticles in natural waters. Environ. Sci. Technol. 46 (16), 9120-9128.

de Laat, W.L., Jaspers, N.G.J., Hoeijmakers, J.H.J., 1999. Molecular mechanism of nucleotide excision repair. Genes Dev. 13 (7), 768-785.

Duran, N., Marcato, P.D., De Conti, R., Alves, O.L., Costa, F.T.M., Brocchi, M., 2010 Potential use of silver nanoparticles on pathogenic bacteria, their toxicity and possible mechanisms of action. J. Braz. Chem. Soc. 21 (6), 949-959.

Duran, N., Duran, M., de Jesus, M.B., Seabra, A.B., Favaro, W.J., Nakazato, G., 2016. Silver nanoparticles: a new view on mechanistic aspects on antimicrobial activity. Nanomed. Nanotechnol. Biol. Med. 12 (3), 789-799.

Fan, X.J., Xu, J.H., Lavoie, M., Peijnenburg, W.J.G.M., Zhu, Y.C., Lu, T., Fu, Z.W., Zhu, T.H., Qian, H.F., 2018. Multiwall carbon nanotubes modulate paraquat toxicity in Arabidopsis thaliana. Environ. Pollut. 233, 633-641.

Gil-Allue, C, Schirmer, K. Tlili, A., Gessner, M.O, Behra, R., 2015. Silver nanoparticle effects on stream periphyton during short-term exposures. Environ. Sci. Technol. 49 (2), 1165-1172.

Gong, W.D., Browne, J., Hall, N., Schruth, D., Paerl, H., Marchetti, A., 2017. Molecular insights into a dinoflagellate bloom. ISME J. 11 (2), 439-452.

Graham, C.M., Ehrenshaft, M., Hausner, G., Reid, D.M., 2004. A highly conserved gene for vitamin B-6 biosynthesis may have consequences for stress and hormone responses in plants. Physiol. Plant. 121 (1), 8-14.

Harper, S.J., LoGrasso, P., 2001. Signalling for survival and death in neurones - the role of stress-activated kinases, JNK and p38. Cell. Signal. 13 (5), 299-310.

Hasegawa, S., Imai, M., Yamasaki, M., Takahashi, N., Fukui, T., 2018. Transcriptional regulation of acetoacetyl-CoA synthetase by Sp1 in neuroblastoma cells. Biochem. Biophys. Res. Commun. 495 (1), 652-658.

Inskeep, W.P., Bloom, P.R., 1985. Extinction coefficients of chlorophyll a and b in N, N-dimethylformamide and 80\% acetone. Plant Physiol. 77, 483-485.

Jiang, H.S. Yin, L.Y., Ren, N.N., Xian, L., Zhao, S.T., Li, W., Gontero, B., 2017. The effect of chronic silver nanoparticles on aquatic system in microcosms. Environ. Pollut. 223, 395-402.

Joo, S.H., Zhao, D.Y., 2017. Environmental dynamics of metal oxide nanoparticles in heterogeneous systems: a review. J. Hazard Mater. 322, 29-47.

Kaegi, R., Sinnet, B., Zuleeg, S., Hagendorfer, H., Mueller, E., Vonbank, R., Boller, M., Burkhardt, M., 2010. Release of silver nanoparticles from outdoor facades. Environ. Pollut. 158 (9), 2900-2905.

Ke, M., Qu, Q., Peijnenburg, W.J.G.M., Li, X., Zhang, M., Zhang, Z., Lu, T., Pan, X., Qian, H., 2018. Phytotoxic effects of silver nanoparticles and silver ions to Arabidopsis thaliana as revealed by analysis of molecular responses and of metabolic pathways. Sci. Total Environ. 644, 1070-1079.

Li, X.X., Ke, M.J., Zhang, M., Peijnenburg, W.J.G.M., Fan, X.J., Xu, J.H., Zhang, Z.Y., Lu, T., Fu, Z.W., Qian, H.F., 2018. The interactive effects of diclofop-methyl and silver nanoparticles on Arabidopsis thaliana: growth, photosynthesis and antioxidant system. Environ. Pollut. 232, 212-219.

Lin, D.H., Ma, S., Zhou, K.J., Wu, F.C., Yang, K., 2015. The effect of water chemistry on homoaggregations of various nanoparticles: specific role of $\mathrm{Cl}^{-}$ions. J. Colloid Interface Sci. 450, 272-278.

Liu, S.Q., Wang, C., Hou, J., Wang, P.F., Miao, L.Z., Li, T.F., 2018a. Effects of silver sulfide nanoparticles on the microbial community structure and biological activity of freshwater biofilms. Environ. Sci. Nano 5, 2899-2908.

Liu, S.Q., Wang, C., Hou, J., Wang, P.F., Miao, L.Z., Fan, X.L., You, G.X., Xu, Y., 2018b. Effects of $\mathrm{Ag}$ and $\mathrm{Ag}_{2} \mathrm{~S}$ nanoparticles on denitrification in sediments. Water Res. $137,28-36$.

Lu, T, Ke, MJ, Peijnenburg WJ.G.M. Zhu, Y.C, Zhang M. Sun, LW. Fu, ZW, Qian, H.F., 2018. Investigation of rhizospheric microbial communities in wheat, barley, and two rice varieties at the seedling stage. J. Agric. Food Chem. 66 (11), 2645-2653.

Lu, T., Zhu, Y., Ke, M., Peijnenburg, W.J.G.M., Zhang, M., Wang, T.Z., Chen, J., Qian, H.F., 2019a. Evaluation of the taxonomic and functional variation of freshwater plankton communities induced by trace amounts of the antibiotic ciprofloxacin. Environ. Int. 126, 268-278.

Lu, T., Zhang, Q., Lavoie, M., Zhu, Y., Ye, Y., Yang, J., Paerl, H., Qian, H., Zhu, Y.G., 2019b. The fungicide azoxystrobin promotes freshwater cyanobacterial dominance through altering competition. Microbiome 7,128.

Luo, H.W. Law, W.W., Wu, Y.C., Zhu, W.P., Yang, E.H., 2018. Hydrothermal synthesis of needle-like nanocrystalline zeolites from metakaolin and their applications for efficient removal of organic pollutants and heavy metals. Microporous Mesoporous Mater. 272, 8-15.

Mansilla, M.C., Albanesi, D., de Mendoza, D., 2000. Transcriptional control of the sulfur-regulated cysH operon, containing genes involved in L-cysteine biosynthesis in Bacillus subtilis. J. Bacteriol. 182, 5885-5892.

Maurer-Jones, M.A. Gunsolus, IL. Murphy, C.J., Haynes, C.L. 2013. Toxicity of engineered nanoparticles in the environment. Anal. Chem. 85 (6), 3036-3049.

Metreveli, G., Philippe, A., Schaumann, G.E., 2015. Disaggregation of silver nanoparticle homoaggregates in a river water matrix. Sci. Total Environ. 535, 35-44.

Meyer, E.H., Tomaz, T., Carroll, A.J., Estavillo, G., Delannoy, E., Tanz, S.K., Small, I.D., Pogson, B.J., Millar, A.H., 2009. Remodeled respiration in ndufs4 with low phosphorylation efficiency suppresses arabidopsis germination and growth and alters control of metabolism at night. Plant Physiol. 151 (2), 603-619.

Nelson, N., Ben-Shem, A., 2004. The complex architecture of oxygenic photosynthesis. Nat. Rev. Mol. Cell Biol. 5 (12), 971-982.

Park, H.G., Yeo, M.K., 2016. Nanomaterial regulatory policy for human health and environment. Mol. Cell. Toxicol. 12 (3), 223-236,

Peters, R.J.B., van Bemmel, G., Milani, N.B.L., den Hertog, G.C.T., Undas, A.K., van der Lee, M., Bouwmeester, H., 2018. Detection of nanoparticles in Dutch surface waters. Sci. Total Environ. 621, 210-218.

Qian, H.F., Peng, X.F., Han, X., Ren, J., Sun, L.W., Fu, Z.W., 2013. Comparison of the toxicity of silver nanoparticles and silver ions on the growth of terrestrial plant model Arabidopsis thaliana. J. Environ. Sci. 25 (9), 1947-1955.

Qian, H.F., Zhu, K., Lu, H.P., Lavoie, M., Chen, S., Zhou, Z.J., Deng, Z.P., Chen, J., Fu, Z.W. 2016. Contrasting silver nanoparticle toxicity and detoxification strategies in Microcystis aeruginosa and Chlorella vulgaris: new insights from proteomic and physiological analyses. Sci. Total Environ. 572, 1213-1221.

Reidy, B., Haase, A., Luch, A., Dawson, K.A., Lynch, I., 2013. Mechanisms of silver nanoparticle release, transformation and toxicity: a critical review of current knowledge and recommendations for future studies and applications. Materials $6(6), 2295-2350$.

Schaumann, G.E., Baumann, T., Lang, F., Metreveli, G., Vogel, H.J., 2015. Engineered nanoparticles in soils and waters Foreword. Sci. Total Environ. 535, 1-2.

Shi, Y.G., Massague, J., 2003. Mechanisms of TGF-beta signaling from cell membrane to the nucleus. Cell 113 (6), 685-700.

Shweta, Tripathi, D.K., Singh, S., Singh, S., Dubey, N.K., Chauhan, D.K., 2016. Impact of nanoparticles on photosynthesis: challenges and opportunities. Mater. Focus 5 (5), 405-411.

Tang, J., Wu, Y.H., Esquivel-Elizondo, S., Sorensen, S.J., Rittmann, B.E., 2018. How 
microbial aggregates protect against nanoparticle toxicity. Trends Biotechnol. 36 (11), 1171-1182.

Xie, J., Bai, X.C., Lavoie, M., Lu, H.P., Fan, X.J., Pan, X.L., Fu, Z.W., Qian, H.F., 2015 Analysis of the proteome of the marine diatom phaeodactylum tricornutum exposed to aluminum providing insights into aluminum toxicity mechanisms. Environ. Sci. Technol. 49 (18), 11182-11190.

Xun, H.W., Ma, X.T., Chen, J., Yang, Z.Z., Liu, B., Gao, X., Li, G., Yu, J.M., Wang, L., Pang, J.S., 2017. Zinc oxide nanoparticle exposure triggers different gene expression patterns in maize shoots and roots. Environ. Pollut. 229, 479-488.

Zhang, C.Q., Hu, Z.Q., Deng, B.L., 2016. Silver nanoparticles in aquatic environments: physiochemical behavior and antimicrobial mechanisms. Water Res. 88, 403-427.

Zhang, M., Yang, J.H., Cai, Z.X., Feng, Y.D., Wang, Y.F., Zhang, D.Y., Pan, X.L., 2019.
Detection of engineered nanoparticles in aquatic environments: current status and challenges in enrichment, separation, and analysis. Environ. Sci. Nano 6 (3), 709-735.

Zheng, Y.L., Hou, L.J., Liu, M., Newell, S.E., Yin, G.Y., Yu, C.D., Zhang, H.L., Li, X.F., Gao, D.Z., Gao, J., Wang, R., Liu, C., 2017. Effects of silver nanoparticles on nitrification and associated nitrous oxide production in aquatic environments. Sci. Adv. 3 (8), e1603229.

Zheng, K.Y., Setyawati, M.I., Leong, D.T., Xie, J.P., 2018. Antimicrobial silver nanomaterials. Coord. Chem. Rev. 357, 1-17.

Zhu, H.H., Ai, H.L., Cao, L.W., Sui, R., Ye, H.P., Du, D.Y., Sun, J., Yao, J., Chen, K., Chen, L. 2018. Transcriptome analysis providing novel insights for Cd-resistant tall fescue responses to Cd stress. Ecotoxicol. Environ. Saf. 160, 349-356. 